\title{
Babesiosis in a Calf: A Case Report
}

\author{
Chigozie S. Ukwueze ${ }^{* 1}$,Chioma F. Orajaka ${ }^{2}$ \\ ${ }^{1}$ (Department of Veterinary Medicine) \\ ${ }^{2}$ (Veterinary Teaching Hospital) \\ Michael Okpara University of Agriculture, Umudike.Abia State.
}

\begin{abstract}
Babesiosis or Tick fever is economically the most important arthropod-borne disease of cattle worldwide. The causative agent is protozoan parasite of the genus Babesia. The two main species known to affect cattle are Babesiabovis and Babesiabigemina. In this case report, the clinical signs observed were; anorexia, emaciation and loss of weight, slight pale mucus membrane, presence of ectoparasites (ticks), and enlarged mandibular and superficial lymph nodes. The peripheral blood smear revealed the presence of piroplasmic organisms Babesia in RBC. The haematology revealed, packed cell volume (PCV) $30 \%$ and haemoglobin concentration (HB) $9.2 \mathrm{~g} / \mathrm{dl}$. The animal was treated with a single dose of Diminazeneaceturate (berenil) IM, at $5 \mathrm{mg} / \mathrm{kg} \mathrm{BW}$, with supportive therapy.
\end{abstract}

Key words: Arthropod-borne, Bovine Babesiosis, Calf, Clinical signs,Diminazeneaceturate.

\section{Introduction}

Bovine babesiosis (BB) is an intraerythrocytic tick borne disease of cattle and buffaloes caused by the protozoan parasites of the genus Babesia [1]. The two main speciesknown to affect cattle are Babesiabovis and Babesiabigemina. The disease is principally transmitted by one host tick Boophilus species. Transmissionoccurtransovariallybetween tick generations [2], by fomites and mechanically from contaminated blood [3]. In utero transmissions have been reported, but are infrequently encountered in calves [4]. Bovine Babesiosis is found in areas where its arthropod vectors are distributed, especially tropical and subtropical climates. B. bovis and B. bigemina are widely distributed and a major importance in Africa, Asia, Australia, Central and South America. B. divergens is economically important in some parts of Europe and North America [1].

The disease is of major economic important and will cost the agricultural sector some colossal amount of money in countries afflicted by this disease [5] as a result of the infection causing retarded growth in calves, death, increased abortion rate and sterility, reduced milk and meat production and escalated cost of prevention and treatments [6].

Babesiosis is the second most widespread blood-borne disease of animals [7,8,2], and prominently, is gaining increasing interest as an emerging zoonosis of humans [7, 9, 10, 8, 11, 2]. Clinically, the disease is characterized by fever and intravascular hemolysis manifested by a syndrome of anaemia, hemoglobinuria and jaundice. The signs can also vary depending on the specie of parasite and the host factor such as age and immune status. BB is predominantly observed in adult cattle, with B. bovis generally being more pathogenic than B. bigemina and B. divergens. Infected animals develop lifelong immunity against re-infection with the same species and cross-protection is evident in B. bigemina- immune animals against subsequent B. bovis infections [2]. BB is also known as tick fever, Texas fever, red water fever [6 12, 13, 2]. Incubation period is often 2-3 weeks or longer after tick infestation. Shorter incubation periods have however been documented in the field and through experimental inoculation (4-5 days for B. bigeminaand 10-12 days for B. bovis)

Babesiosis is particularly severe in naive animals introduced into endemic areas and is a considerable constraint on livestock development in many parts of the world. BB is principally maintained by sub-clinically infected cattle that have recovered from the disease.Morbidity and mortality vary greatly and are influenced by prevailing treatments employed in an area, previous exposure to a species/strain of parasite and vaccination status. In endemic areas, cattle become infected at a young age and develop a long-term immunity. However, outbreaks can occur in these endemic areas if exposure to ticks by young animals is interrupted or immunonaive cattle are introduced. The introduction of Babesiainfected ticks into previously tick-free areas may also lead to outbreaks of disease. Typically, Babesia organisms are pyriform, but may be round, elongated or cigar shaped.

\section{Case History}

A two month old calf was presented to the Veterinary Teaching Hospital, Michael Okpara University of Agriculture, Umudike with a history of anorexia, emaciation and loss of weight. On clinical examination the 
rectal temperature was $38.1^{\circ} \mathrm{C}$, slight pale mucus membrane, presence of ectoparasites (ticks), and enlarged mandibular and superficial lymph nodes (Figures $1 \& 2$ ).

Blood was collected from the jugular vein into EDTA bottle to check for haemoparasites and some haematological parameters. The thin smear stained with Giemsa revealed the presence of piroplasmic organismsBabesia in RBC. The haematology revealed, packed cell volume (PCV) $30 \%$ and haemoglobinconcentration (HB) $9.2 \mathrm{~g} / \mathrm{dl}$.

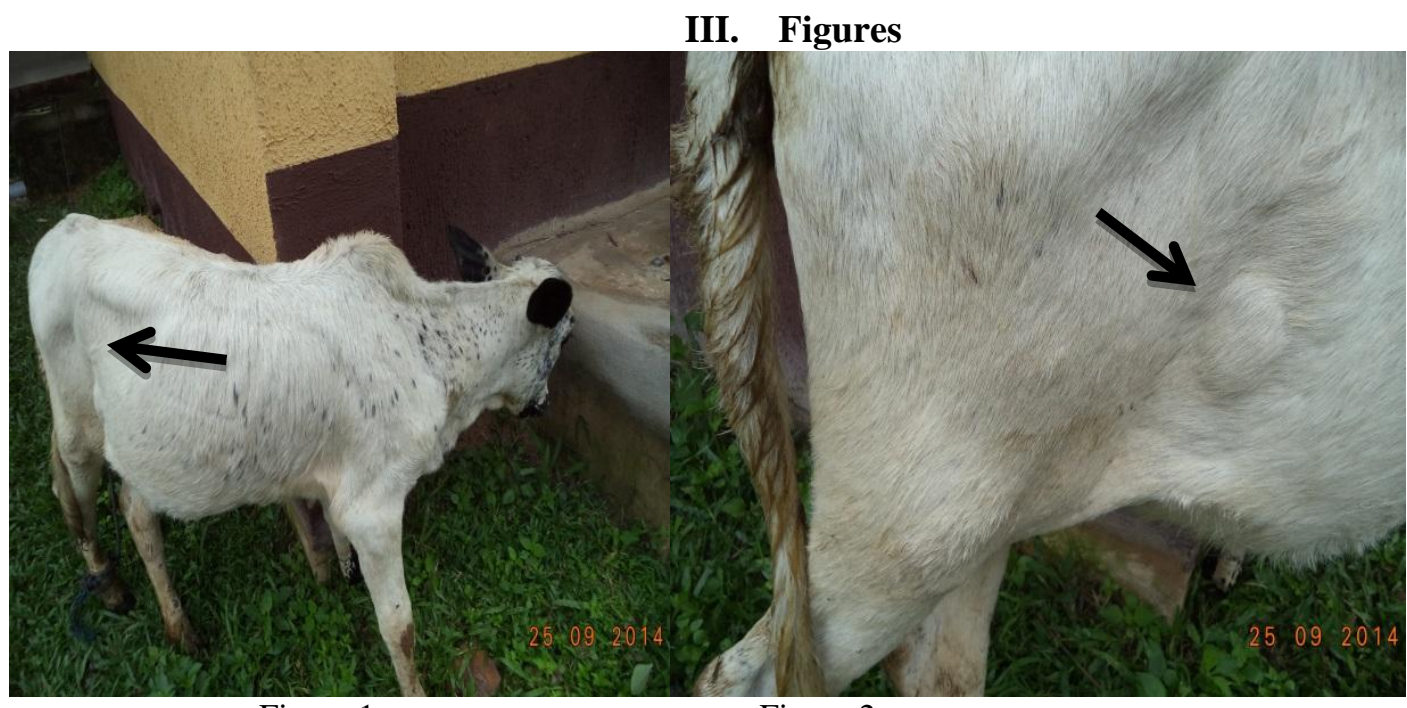

Figure 1

Figure 2

\section{Results and Discussion}

Based on clinical signs and laboratory examination the condition was diagnosed to be bovine babesiosis.The animal was treated with diminazeneaceturate(Berenil) at $5 \mathrm{mg} / \mathrm{kg} \mathrm{BW}$, IM and Ivermectin at 0.2 $\mathrm{mg} / \mathrm{kg} \mathrm{SC}$, pour on was applied topically on the skin at the dorsal aspect from the head down to the tail region. Berenil is an antiprotozoal drug for treatment and control of protozoa infection in cattle, sheep, horses and dogs. It also protects cattle against Babesiosis and Trypanosomiasis for 2-4 weeks. Ivermectin was administered for the control of endo and ectoparasites. The calf was also administered with vitaflash (multivitamin) $5 \mathrm{ml}$ IM, as a supplement for iron and vitamin to increase RBC formation. The owner was advised to present the animal after one week for further examination. After one week, the peripheral blood smear examination was found to be negative for Babesiaorganisms. Haematological analysis revealed PCV andhaemoglobinconcentration values within the normal range. The animal showed marked improvement and eventually recovered.Karunakaran et al. [14] successfully treated a twenty day old calf with a single dose of diminazeneaceturate and supportive therapy.

In endemic areas of bovine babesiosis calves develop a degree of immunity, related both to colostralderived antibodies and age that persists for about 6 months. At high levels of tick transmission, all newborn calves will become infected with Babesiaby 6 months of age, show few if any clinical signs, and subsequently developimmunity. The immune response of cattle to B. bovisor B. bigemina infection involves both innate and acquired immune mechanisms. Innate immunity is non-specific and includes factors such as host-parasite specificity, genetic factors, age of the host and the response of host cells. Trueman and Blight[15] and Goff et al. [16] stated that young calves exhibit a strong innate immunity compared to adult cattle. Infected animals with most Babesia speciesdevelop the immunity against reinfection with the same species.Cattle with the history and clinical signs of fever, anemia, jaundice and haemoglobinuria should be suspected for Babesiosis. However, the differential diagnosis comprises of Babesiosis, Trypanosomiasis, Anaplasmosis, Leptospirosis and Theileriosis. The disease can be diagnosed by the examination of blood and organ smears with Giemsa. From the live animal, thick and thin blood smears should be prepared, preferably from capillaries in the ear or tail tip. Thick films can be helpful in detecting small numbers of parasites, but species identification is best in thin films. At necropsy, smears of heart muscle, kidney, liver, lung, and brain and from a blood vessel in the extremities (lower legs) should be taken. Diagnosis is unreliable in cattle that have died for more than 24 hours.

Post mortem lesions are mainly related to intravascular hemolysis, anaemia and jaundice. The mucous membrane is usually pale and may be icteric. The subcutaneous tissue may also be icteric. The spleen is markedly enlarged with a dark, pulpy and friable consistency. The liver may be enlarged and darkened or icteric with a distended gallbladder containing thick, granular bile. The kidneys are usually dark red or black, and the urinary bladder often contains reddish-brown urine. However, in some cases, the urine may be normal. Other 
organs may show congestion or petechial haemorrhages and occasionally there will be pulmonary edema $[17$, $18]$.

The morbidity and mortality rates are highly variable. Cattle can develop lifelong resistance to a species after infection. Animals that recover from natural infection remain immune with persistence of the parasite in the peripheral blood for a number of years with B. bovis and for a few months in the case of $B$. bigemina. This phenomenon is termed pre munity. No signs are apparent during this carrier state but protection can be broken down by stress factors such as parturition, starvation, or concurrent diseases and clinical signs may reappear. Repeated infections results in permanent immunity. If the illness is treated urgently and efficiently, and the protozoa are killed before antibodies are produced, no immunity occurs [19].

Imidocarbdipropionate is the drug of choice to treat bovine babeisois followed by Dimenazineaceturate, with 100 per cent and 90 per cent efficacy at 10 day post treatment respectively [20]. In the present case, the animal showed good response to Dimenazineaceturate 7 days post treatment as evident from the blood picture. Control measures that are currently applied are control of ticks by use of acaricides, vaccination and chemoprophylaxis. The most effective procedure for the control of babesiosis is to control and eradicate its vector, the Boophilus tick. It can be by dipping or regular spraying every 2-3 weeks. The common acaricides used for combating ticks are the chlorinated hydrocarbons, carbamates, natural and synthetic pyrethrins, and avermectins. Live, attenuated strains of B. bovis, B. bigeminaor B. divergensare used to vaccinate cattle.

[1] OIE, Bovine babesiosis in OIE Update, 2013

\section{References}

[2] S. Gohil, H. Susann, G. Svenja, and M.C. Brian, Bovine babesiosis in the 21st century: Advances in biology and functional genomics. International Journal for Parasitology. 43, 2013, 125-132.

[3] F.F.J. Abdullah, L. Adamu, A.Y. Osman, A.W. Haron, and A.A. Sharee, Clinical management of an outbreak of babesiosis in a herd of cattle: A case report. Journal of Agricultural and Veterinary science. 4, 2013, 78-83.

[4] W.K. Jorgensen, The Merck Veterinary Manual 9th Edn. The Merck \& Co Inc, Whitehouse Station, NJ, USA. $2008,21-26$

[5] D. Sackett, P. Holmes, K. Abbott, S. Jephcott, and M. Barber, Assessing the economic cost of endemic disease on the profitability of Australian beef cattle and sheep producers. Meat \& Livestock Australia, Sydney. Limited Final Report, AHW.087, 2006. Available from: <http://www.mla.com.au/Research-anddevelopment/Final-report details project id=3578>.

[6] R. Bock, L. Jackson, A. de Vos, and W. Jorgensen, Babesiosis of cattle. VeterinaryParasitology 129, 2004, S247-S269.

[7] M.J. Homer, I. Aguilar-Delfin, S.R. Telford III, P.J. Krause, and D.H. Persing, Babesiosis. Clinical Microbiology Rev. 13, 2000, 451-469.

[8] K.P. Hunfeld, A. Hildebrandt, and J.S. Gray, Babesiosis: recent insights into an ancient disease. Int. J. Parasitol. 38, 2008, 12191237.

[9] A.M. Kjemtrup, and P.A. Conrad, Human babesiosis: an emerging tick-borne disease. Int. J. Parasitol. 30, $2000,1323-1337$.

[10] A. Zintl, G. Mulcahy, H.E. Skerrett, S.M. Taylor, and J.S. Gray, Babesiadivergens bovine blood parasite of veterinary and zoonotic importance.Clin.Microbiol. Rev. 16, 2003, 622-636.

[11] D.A. Leiby, Transfusion-transmitted Babesia spp.: bull's-eye on Babesiamicroti. Clin.Microbiol. Rev. 24, 2011, 14-28.

[12] B.M. Cooke, N. Mohandas, A.F. Cowman, and R.L. Coppel, Cellular adhesive phenomena in apicomplexan parasites of red blood cells. Veterinary Parasitology. 132, 2005, 273-295.

[13] S. Gohil, L.M. Kats, A. Sturm, and B.M. Cooke, Recent insights into alteration of red blood cells by Babesiabovis: moovin' forward. Trends Parasitology. 26, 2010, 591-599.

[14] S. Karunakaran, U. Narayana, A.M. Kurisakose, G. Aswathy, K.T. Rajimon, and M. Sumangala.Babesiabigemina infection in twenty day old calf: clinical report. JIVA 9: (1) 2011, 49-50.

[15] K.F. Trueman, and G.W. Blight, The effect of age on resistance of cattle to Babesiabovis.AustralianVeterinary Journal, 54, 1978, 301-305.

[16] W.L. Goff, W.C. Johnson, S.M. Parish, G.M. Barrington, W. Tuo, and R.A. Valdez, The age related immunity in cattle to Babesiabovis infection involves the rapid induction of interleukin-12, interferon-gamma and inducible nitric oxide synthase mRNA expression in the spleen. Parasite Immunology, 23, 2001, 463-471.

[17] M.G. Fincher, W.J. Gibbons, K. Mayer, and S.E. Park, Cattle Tick fever, Diseases of Cattle, 2001, 667-674.

[18] M.K. Cynthia, The Merck Veterinary Manual 9th Edition. Merck and Co. Inc. 2005.

[19] G.M. Urquhart, J. Armour, J.L. Dungan, A.M. Dunn, and F.W. Jennings, (1996): 2nd Edition, Veterinary Parasitology, 1996, 242245.

[20] N. Niazi, M.S. Khan, M. Avais, J.A. Khan, and M. Ijaz, Pakistan Journal of Agricultural Science.45, 2008. 